\title{
THE PRIME RADICAL IN SPECIAL JORDAN RINGS
}

\author{
BY \\ T. S. ERICKSON AND S. MONTGOMERY
}

\begin{abstract}
If $R$ is an associative ring, we consider the special Jordan ring $R^{+}$, and when $R$ has an involution, the special Jordan ring $S$ of symmetric elements. We first show that the prime radical of $R$ equals the prime radical of $R^{+}$, and that the prime radical of $R$ intersected with $S$ is the prime radical of $S$. Next we give an elementary characterization, in terms of the associative structure of $R$, of primeness of $S$. Finally, we show that a prime ideal of $R$ intersected with $S$ is a prime Jordan ideal of $S$.
\end{abstract}

I. Introduction. Using constructions of I. N. Herstein, Kevin McCrimmon [5] has related the Jacobson radical of an associative ring $R$ to the Jacobson radical of the special Jordan ring $R^{+}$, and when $R$ has an involution, to the Jacobson radical of the Jordan ring $S$ of symmetric elements of $R$. More specifically, he has shown:

(1) The Jacobson radical of $R^{+}$coincides with the Jacobson radical of $R$.

(2) The Jacobson radical of $S$ coincides with the Jacobson radical of $R$ intersected with $S$.

In this paper we first prove analogous results for the prime radical (or lower nil radical) of $R$. Then, using these theorems, we give an elementary characterization, in terms of the associative structure of a ring with involution, of primeness of the Jordan ring of symmetric elements. Finally, we examine the relationships between prime ideals of $R, R^{+}$, and $S$.

The methods of proof differ from McCrimmon's since we use absolute zero divisors (see definition below) in special Jordan rings. Absolute zero divisors were examined by N. Jacobson [2] to obtain a structure theorem for semisimple Jordan algebras satisfying descending chain conditions on inner ideals. McCrimmon [4] extended these results to semisimple quadratic Jordan algebras (with the same chain conditions) with no restriction on the characteristic. Their results are analogous to the Artin-Wedderburn theorems for associative rings; however, in the Jordan theory, the concept of semisimplicity was expressed in terms of the absence of nonzero absolute zero divisors rather than in terms of a radical being zero. McCrimmon [6] has since defined the Jacobson radical for quadratic Jordan algebras, and has shown that with the chain conditions mentioned above, the

Presented in part to the Society, January 23, 1970 under the title Jordan systems with involution. II; received by the editors July $28,1970$.

AMS 1970 subject classifications. Primary 17C50, 16A66; Secondary 16A28, 16A68, 16 A21.

Key words and phrases. Special Jordan ring, prime ring, prime ideals, prime radical, lower nil radical. 
absence of absolute zero divisors is equivalent to the Jacobson radical being zero. In associative rings, it is well known that the absence of Jordan absolute zero divisors is equivalent to the prime radical being zero. We will show that, in an associative ring with involution, $S$ has no absolute zero divisors if and only if the prime radical of $S$ is zero. No chain conditions will be imposed.

Since we will discuss arbitrary rings, there are two possible approaches we may take in considering $R^{+}$as a Jordan ring. The more traditional approach, as in the work of Herstein, is to define the Jordan product by $x \circ y=x y+y x$. Alternatively, we could use the quadratic Jordan ring of McCrimmon, with the quadratic operator $x U_{y}=y x y$ and the binary composition $x^{2}$. The major difference between these definitions is that the notion of ideal in a quadratic Jordan ring is more restrictive: using $x \circ y=x y+y x$, an ideal $I$ of $R^{+}$is simply an additive subgroup of $R$ closed under $x \circ y$, where $x \in I, y \in R$; but in a quadratic Jordan ring an ideal $I$ must, in addition, be closed under $x U_{y}, y U_{x}$, and $x^{2}$, where $x \in I, y \in R$.

Except for several cases in which stronger results may be obtained for quadratic Jordan rings, the theorems in this paper are valid for both types of Jordan rings. However, to simplify the presentation we will assume only that ideals are closed under $x \circ y$, unless otherwise specified. In the instances in which we consider $R$ only as a quadratic Jordan ring, we will use the notation " $R^{q}$ " instead of $R^{+}$.

Finally, it should be noted that the only properties of $S$ that will be used are (1) $r+r^{*} \in S$, (2) $r s r^{*} \in S$, and (3) $s^{2} \in S$ for $r \in R, s \in S$. Thus, everything proven for $S$ will actually hold for any subring $T$ satisfying (1) through (3) above. This fact will be needed in §III to state another version of Theorem 7.

The definitions of semiprime and prime Jordan ideals are due to Chester Tsai [7]. In the following, $J$ is a Jordan ring and ideals of $J$ are Jordan ideals.

(1) $P$ is a prime ideal of $J$ if $A U_{B} \subseteq P$, where $A$ and $B$ are ideals of $J$, implies that $A \subseteq P$ or $B \subseteq P$.

(2) $J$ is a prime Jordan ring if (0) is a prime ideal.

(3) $Q$ is a semiprime ideal of $J$ if $A U_{A} \subseteq Q$, where $A$ is an ideal of $J$, implies that $A \subseteq Q$.

(4) The prime radical $P(J)$ of $J$ is the intersection of the prime ideals.

(5) $J$ is a semiprime Jordan ring if $(0)$ is a semiprime ideal of $J$.

THEOREM (TSAI). The intersection of the semiprime ideals of $J$ coincides with the intersection of the prime ideals. Thus, $J$ is semiprime if and only if $P(J)=(0)$.

Although Tsai's proof of this theorem is for classical Jordan algebras, it is easy to see that the same proof works for our special Jordan rings discussed above.

The element $a \in J$ is said to be an absolute zero divisor if $J U_{a}=(0)$; that is, if $a j a=0$, all $j \in J$. We make the following definition: $Q$ is a strongly semiprime ideal of $J$ if $J U_{a} \subseteq Q$ implies that $a \in Q$.

Definition (MCCRimmon). The lower radical $L(J)$ of a Jordan ring $J$ is the intersection of the strongly semiprime ideals of $J$. 
$R$ will always denote an associative ring; if $R$ has an involution $*, S$ will be the set of symmetric elements of $R$. The prime radical (=lower nil radical) of $R$ will be denoted by $N$. We will also say that $R$ is semiprime if $R$ contains no nonzero nilpotent ideals, and that $Q$ is a semiprime ideal of $R$ if $R / Q$ is semiprime.

II. The prime radical and semiprime ideals. In this section, we will investigate the relationship between the prime radical of $R$ and the prime radicals of $R^{+}, R^{q}$, and $S$. We will also investigate the relationship between the lower radical and the prime radical of $S$. We first prove a theorem which is valid for any quadratic Jordan ring.

THEOREM 1. Let $J$ be a quadratic Jordan ring and $Q$ be a quadratic ideal of $J$. If $Q$ is a strongly semiprime ideal of $J$, then $Q$ is a semiprime ideal of $J$.

Proof. Suppose $A$ is a quadratic ideal of $J$ such that $A U_{A} \subseteq Q$. Since $A$ is a quadratic ideal of $J, t U_{a} U_{s} \in A$ for $a \in A$, and $s, t \in J$. Therefore, $t U_{a} U_{s} U_{a} \in Q$. But $t U_{a} U_{s} U_{a}=t U_{s U_{a}}$ by the fundamental identity for quadratic Jordan rings. Since $Q$ is strongly semiprime and $t$ is arbitrary, $s U_{a} \in Q$, which, in turn, implies $a \in Q$ by the same reasoning. Thus, $A \subseteq Q$, and $Q$ is a semiprime ideal of $J$.

COROLlaRY. If $J$ is a quadratic Jordan ring, then $L(J) \supseteq P(J)$.

In the remainder of this section, $R$ will be an associative ring with involution *, and $S$ the symmetric elements of $R$. We will now focus on showing that the prime radical of $S$ coincides with the prime radical $N$ of $R$ intersected with $S$. As a first step, we prove a theorem relating semiprime ideals in $R$ to semiprime ideals of $S$.

THEOREM 2. If $Q$ is a semiprime ideal of $R$, then $Q \cap S$ is a strongly semiprime ideal of $S$ and is a semiprime ideal of $S$.

Proof. $Q \cap S$ is obviously an ideal of $S$. Suppose $a \in S$ and $a S a \subseteq Q \cap S$. We want to show $a \in Q$. Let $x$ be any element of $R$. Then $x^{*} x^{*} \in S$ so that axax*a $\in Q \cap S$. Also $x+x^{*} \in S$ so that $a\left(x+x^{*}\right) a=a x a+a x^{*} a \in Q \cap S$. Write axa

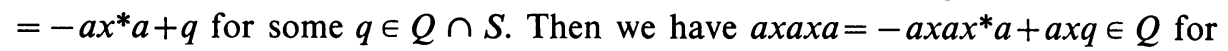
any $x \in R$. Linearizing on $x$, axaya +ayaxa $\in Q$ for all $x, y \in R$. Multiplying by $x a$ on the right gives axayaxa+ayaxaxa $\in Q$. Since ay $($ axaxa $) \in Q$, we have axayaxa $\in$. But $Q$ is a semiprime ideal of $R$; thus, axa $Q$ for all $x \in R$, which, in turn, implies $a \in Q \cap S$. Therefore, $Q \cap S$ is a strongly semiprime ideal of $S$.

To show that $Q \cap S$ is a semiprime ideal of $S$, we assume $A$ is an ideal of $S$, such that $A U_{A} \subseteq Q \cap S$. Thus, $a b a \in Q \cap S$ for all $a, b \in A$ and $a^{3} \in Q \cap S$ for all $a \in A$.

Now $a s+s a \in A$ for $s \in S$, so $a(a s+s a) a \in Q \cap S$. $a^{2} s a+a s a^{2} \in Q \cap S$. Thus, $\left(a^{2} s a+a s a^{2}\right) a \in Q$. But $a^{3} \in Q$, so $a s a^{3} \in Q$, and we have $a^{2} s a^{2} \in Q \cap S$. Since $Q \cap S$ is strongly semiprime, we have $a^{2} \in Q \cap S$. Now $(a s+s a)^{2}=a s a s+s a s a$ $+a s^{2} s+a s^{2} a \in Q \cap S$, so $(a s+s a)^{2} a \in Q$. Since $a^{2} \in Q \cap S$, sasa $a^{2}, s a^{2} s a$, and $a s^{2} a^{2} \in Q$. Thus, asasa $Q \cap S$. Linearizing as above, we have asatasa $\in \cap S$. 
But $Q \cap S$ is strongly semiprime in $S$, so asa $\in \cap S$, which, in turn, implies $a \in Q \cap S$.

Note that for quadratic Jordan rings, the last portion of Theorem 2 is unnecessary, since strongly semiprime implies semiprime by Theorem 1 above. Also, it is only necessary to assume that $Q \cap S$ is a strongly semiprime ideal of $S$ in order to do the last portion of Theorem 2 .

LEMMA 1. $N \cap S \subseteq P(S) \cap L(S)$.

Proof. Let $\mathscr{M}$ denote the set of all ideals $M$ such that $M \cap S \subseteq P(S) \cap L(S)$. By Zorn's lemma, we choose a maximal element $M$ in $\mathscr{M}$. Then $M$ is semiprime, for if $(J / M)^{2}=(0)$ in $R / M$, then $J^{2} \subseteq M$. Thus, $(J \cap S) U_{(J \cap S)} \subseteq S U_{(J \cap S)} \subseteq S \cap R U_{J}$ $\subseteq S \cap J^{2} \subseteq S \cap M \subseteq P(S) \cap L(S)$. But $(J \cap S) U_{(J \cap S)} \subseteq P(S)$ implies $J \cap S \subseteq P(S)$ and $S U_{(J \cap S)} \subseteq L(S)$ implies $J \cap S \subseteq L(S)$. Hence $J \in \mathscr{M}$. Since $M$ is maximal in $\mathscr{M}, J=M$ and $M$ is semiprime. Therefore, $N \subseteq M$ and $N \cap S \subseteq M \cap S \subseteq P(S)$ $\cap L(S)$.

Using Theorem 2 and Lemma 1, we are now able to show that $N \cap S=P(S)$ $=L(S)$.

THEOREM 3. If $R$ is an associative ring with involution $*, N$ is the prime radical of $R$ and $S$ is the set of symmetric elements of $R$, then the prime radical and the lower radical of $S$ coincide with $N \cap S: N \cap S=P(S)=L(S)$.

Proof. By Theorem 2, $N \cap S$ is semiprime and strongly semiprime in $S$. But $P(S)$ and $L(S)$ are the smallest such ideals, so $N \cap S \supseteq P(S)$ and $N \cap S \supseteq L(S)$. The reverse inclusions follow easily from Lemma 1.

COROLlARY. $S$ is a semiprime Jordan ring if and only if $S$ has no nonzero absolute zero divisors.

THEOREM 4. If $R^{+}$denotes $R$ as a Jordan ring, then $N=P\left(R^{+}\right)=L\left(R^{+}\right)=P\left(R^{q}\right)$ $=L\left(R^{q}\right)$.

Proof. Let $A=R \oplus R^{0}$, where $R^{0}$ is the opposite ring of $R$, and give $A$ the exchange involution. Then $S_{A}$, the symmetric elements of $A$, are given by $S_{A}=\left\{\left(r, r^{0}\right) \mid r \in R\right\}$. Note that $S_{A} \simeq R^{+}$as Jordan rings.

If $N_{A}$ denotes the lower nil radical of $A$, then by Theorem 3, $P\left(S_{A}\right)=L\left(S_{A}\right)$ $=N_{A} \cap S_{A}=(N \oplus N) \cap S_{A} \simeq N^{+}$. Thus, $P\left(R^{+}\right)=L\left(R^{+}\right)=N$. The same argument holds true for $R^{q}$.

COROLlaRY 1. $R$ is a semiprime associative ring if and only if $R^{+}$is a semiprime Jordan ring if and only if $R^{q}$ is a semiprime quadratic Jordan ring.

COROllaRY 2. Let $Q$ be an ideal of $R$. Then $Q$ is a semiprime ideal of $R$ if and only if $Q^{+}$is a semiprime Jordan ideal of $R^{+}$.

Proof. Note that $(R / Q)^{+} \simeq R^{+} / Q^{+}$as Jordan rings and apply Corollary 1 . 
We conclude this section with an additional result for $R$ as a quadratic Jordan ring.

THEOREM 5. Let $R$ be an associative ring. Then $Q$ is a strongly semiprime quadratic ideal of $R^{q}$ if and only if $Q$ is a semiprime ideal of $R$.

Proof. Suppose $Q$ is a semiprime ideal of $R$; then $Q$ is obviously a quadratic ideal of $R^{a}$. Suppose $a R a \subseteq Q$. Then $a \in Q$ since $Q$ is a semiprime ideal of $R$.

Suppose $Q$ is a strongly semiprime quadratic ideal of $R^{q}$. For $q \in Q$ and $x, y \in R, q(x y)(q x)+(q x)(x y) q \in Q$ since $Q$ is a quadratic ideal of $R^{q}$. But $q(x x y) q \in Q$ for the same reason, so we have $q x y q x \in Q$. Since $Q$ is strongly semiprime, $q x \in Q$ and, thus, $Q$ is a right ideal of $R$. Similarly, $x q y x q \in Q$ and $x q \in Q$, so $Q$ is an ideal of $R$. Suppose $a R a \subseteq Q$. Then $a \in Q$. Therefore, $Q$ is a semiprime ideal of $R$.

III. Primeness and prime ideals. In this section, we show that $S$ being a prime Jordan ring is equivalent to the following:

$$
\text { "If } a S b=(0) \text {, where } a, b \in S \text {, then } a=0 \text { or } b=0 \text {." }
$$

Note that this condition looks very much like the condition for an associative ring to be prime.

THEOREM 6. If $R$ is prime, then $a S b=(0), a, b \in S$ implies that $a=0$ or $b=0$.

Proof. We actually prove a somewhat stronger statement, that is, if $a S b=(0)$ where either $a$ or $b$ is in $S$, then $a=0$ or $b=0$.

Say that $a \in S$. Then $x a x^{*} \in S$, so $a x a x^{*} b=0$, for any $x \in R$. Applying *, $b^{*} x a x^{*} a=0$, all $x \in R$. Linearizing on $x$, we have

$$
b^{*} x a y^{*} a+b^{*} y a x^{*} a=0, \quad \text { all } x, y \in R .
$$

Multiplying by $x b$,

$$
b^{*} x a y^{*} a x b+b^{*} y a x^{*} a x b=0, \quad \text { all } x, y \in R .
$$

Since $a x^{*} a x b=0, b^{*} x a R a x b=(0)$, as $y$ was any element of $R$. Since $R$ is prime, it must happen that for each $x \in R$, either $a x b=0$ or $a x^{*} b=0$. But since $x+x^{*} \in S$, $a\left(x+x^{*}\right) b=0$, and so $a x b=-a x^{*} b$. Thus, $a x b=0$, all $x \in R$, so $a R b=(0)$. Using again that $R$ is prime, either $a=0$ or $b=0$.

We now proceed to show that the $a S b$ condition is actually equivalent to $S$ being prime. One direction is fairly easy.

Lemma 2. If $a S b=(0), a, b \in S$ implies $a=0$ or $b=0$, then $S$ is prime.

Proof. Assume that $S$ is not prime, that is, $B U_{A}=(0)$, where $A$ and $B$ are ideals of $S$. Thus, $a b a=0$, for all $a \in A, b \in B$

Say that $B \neq(0)$, and choose $b \neq 0$ in $B$. We will show that $A=(0)$. Consider $A \cap B ; A \cap B$ is an ideal of $S$ and $(A \cap B) U_{(A \cap B)}=(0)$. Since $S$ certainly has no 
absolute zero divisors, $S$ must be semiprime by the corollary to Theorem 3 . Thus, $A \cap B=(0)$, as (0) is a semiprime ideal.

Now for any $a \in A, a b+b a \in A \cap B=(0)$, so $a b=-b a$. Thus, $a^{2} b=-a b a=0$. Since this is true for any $b \in B$, replace $b$ by $b s+s b$, any $s \in S$. Then $a^{2}(b s+s b)=0$ $=a^{2} b s+a^{2} s b=a^{2} s b$. Since $b \neq 0$ and $a^{2} \in S$, by the $a S b$ condition we must have $a^{2}=0$, all $a \in A$.

Let $s$ be any element of $S$. Then $a s+s a \in A$, so $(a s+s a)^{2}=0=a s a s+s a s a+s a^{2} a$ $+a s^{2} a$. Thus, multiplying by $a$ and using that $a^{2}=0$, we see that asasa $a$, all $s \in S$. Linearizing on $s$ gives, as usual, asarasa $=0$, all $r, s \in S$. This says that asa is an absolute zero divisor in $S$, which is impossible. Thus, as $a=0$, all $s \in S$. By the same argument, $a=0$. Thus, $A=(0)$.

LeMmA 3. If $S$ has no absolute zero divisors and $a S b=(0), a, b \in S$, then $a x b x a=0$ and $b x a x b=0$, all $x$ in $R$.

Proof. We show only that $a x b x a=0$, as the proof that $b x a x b=0$ is exactly the same.

Since $x+x^{*} \in S, a x b=-a x^{*} b$, all $x \in R$. Also, $x a x^{*} \in S$, so $a x a x^{*} b=0$. Thus, $a x a x b=-a x a x^{*} b=0$, all $x \in R$. Applying *, we see that also bxaxa $=0$. Linearizing on $x$,

$$
\text { bxaya }+ \text { byaxa }=0, \quad \text { all } x, y \in R .
$$

Multiplying by $x b$, since $a x a x b=0$, we have $b x a y a x b=0$. Then $b x a y a x^{*} b=0$, since $a x b=-a x^{*} b$, and, thus, $\left(a x^{*} b x a\right) S\left(a x^{*} b x a\right)=(0)$. Since $a x^{*} b x a \in S$ and $S$ has no absolute zero divisors, $a x^{*} b x a=0$. But then $a x b x a=0$.

THEOREM 7. $S$ is prime if and only if $a S b=(0)$ for $a, b \in S$ implies $a=0$ or $b=0$.

Proof. From Lemma 2, it is enough to show that if $S$ is prime, then $S$ satisfies the $a S b$ condition. So, assume that $S$ is prime but that $a S b=0$, some $a, b \in S$. By the corollary to Theorem $3, S$ has no absolute zero divisors. By Lemma 3, axbxa $=0$, for any $x \in R$. Linearizing on $x$, and multiplying by $x b$,

$$
\text { axbyaxb }=0, \quad \text { all } x, y \in R .
$$

But then $a x b \in N$, the lower nil radical of $R$, as $a x b R$ is a nilpotent ideal. Applying *, $b x^{*} a \in N$ for any $x \in R$. As a result, $a R b, b R a, R a R b R$, and $R b R a R$ are all contained in $N$.

Let $I=[a]$, the principal Jordan ideal of $S$ generated by $a$, and let $J=[b]$. We will show that $J U_{I}=(0)$.

Note that $a b a=0$, for since $a s+s a \in S, a(a s+s a) b=0=a^{2} s b+a s a b$. Thus, $a S a b=(0)$, so $a b a S a b a=(0)$. Since $S$ has no absolute zero divisors, $a b a=0$.

Now $J U_{I} \subseteq R a b a R+R a R b R+R b R a R \subseteq N$, and since clearly $J U_{I} \subseteq S$, we have $J U_{I} \subseteq N \cap S$. Since $S$ is semiprime, $P(S)=(0)$, so $N \cap S=(0)$ by Theorem 3. Thus, $J U_{I}=(0)$. But now since $S$ is prime, $I=(0)$ or $J=(0)$, so $a=0$ or $b=0$ and we are done. 
COROLlARY. If $R$ is a prime associative ring with ${ }^{*}$, then $S$ is a prime Jordan ring.

Proof. Theorems 6 and 7.

It should be pointed out that the converse of the corollary (and of Theorem 6) is false. For let $R$ be any prime ring and let $A=R \oplus R^{0}$, where $R^{0}$ is the opposite ring of $R$. Give $A$ the exchange involution. Then the symmetric elements of $A$ have the $a S b$ condition, since $R$ is prime, and so $S_{A}$ is prime. However, $A$ is certainly not prime.

As another corollary to Theorem 7, we can now show that $R$ being prime as an associative ring is equivalent to $R^{+}$being prime as a Jordan ring.

THEOREM 8. $R$ is a prime associative ring if and only if $R^{+}$is a prime Jordan ring.

Proof. As in the proof of Theorem 4, we use $A=R \oplus R^{0}$. If $R$ is prime, then $S_{A}$ must be prime. For, say that $\left(a, a^{0}\right)\left(r, r^{0}\right)\left(b, b^{0}\right)=0$ for all $r \in R$. This says that $\left(a r b,(b r a)^{0}\right)=(0,0)=0$, or $a r b=0$, all $r \in R$. Since $R$ is prime, $a=0$ or $b=0$. Thus, $S_{A}$ satisfies the $a S b$ condition, so is prime by Theorem 7. Since $R^{+} \simeq S_{A}$ as Jordan rings, we have that $R^{+}$is prime.

Now assume that $R^{+}$is prime. If $R$ is not prime, then $A B=(0), A, B$ ideals of $R$. But $A$ and $B$ are also ideals of $R^{+}$, and $A U_{B} \subseteq A B=(0)$. Thus, $A=(0)$ or $B=(0)$ since $R^{+}$is prime, and, thus, $R$ itself is prime.

Corollary. Let $P$ be an ideal of $R$. Then $P$ is a prime ideal of $R$ if and only if $P^{+}$is a prime Jordan ideal of $R^{+}$.

Proof. Note that $(R / P)^{+} \simeq R^{+} / P^{+}$as Jordan rings and apply Theorem 8 .

Returning to a ring $R$ with *, we next show that a weaker condition than $R$ being prime is sufficient for $S$ to be prime.

Definition. A ring $R$ with involution * is *-prime if $A B=(0)$ implies $A=(0)$ or $B=(0)$, where $A$ and $B$ are ideals of $R$ with $A^{*}=A$ and $B^{*}=B$.

Note that the ring $A=R \oplus R^{0}$ discussed after Theorem 7 is *-prime even though it is not prime. Also, it is fairly easy to show that any *-prime ring is semiprime [3, p. 193].

As noted at the end of $\S I$, our results are for more general Jordan rings than $S$. A general form of Theorem 7 will be needed in the proof of Theorem 10 .

THEOREM 7'. Let $R$ be an associative ring with * and let $T$ be a Jordan subring of $S$ such that

(1) $x+x^{*} \in T$, for all $x \in R$;

(2) $x t x^{*} \in T$, for any $t \in T$ and $x \in R$;

(3) $t^{2} \in T$, for any $t \in T$.

Then $T$ is a prime Jordan ring if and only if $a T b=(0)$ for $a, b \in T$ implies $a=0$ or $b=0$.

THEOREM 9. If $R$ is $a^{*}$-prime ring with involution * and $T$ satisfies the hypotheses of Theorem $7^{\prime}$, then $T$ is a prime Jordan ring. 
Proof. Assume that $a T b=(0)$, where $a, b \in T$. By Theorem $7^{\prime}$, it will be enough to show that $a=0$ or $b=0$. Since $R$ is semiprime, $T$ has no absolute zero divisors (a simpler form of the proof of Theorem 6 shows this). Thus, by following the computations in Lemma 3, we have $a x b x a=0=b x a x b$, for all $x \in R$. Then, as usual, this implies $a x b y a x b=0$, all $x, y \in R$. Since $R$ is semiprime, this means $a x b=0$, all $x \in R$.

Let $A=R a R$ and $B=R b R$. Certainly $A^{*}=A$ and $B^{*}=B$ since $a, b \in T \subseteq S$, but $A B=(0)$. This contradicts $R$ being *-prime unless $A=(0)$ or $B=(0)$. But then $a=0$ or $b=0$.

COROllaRY. If $R$ is *-prime, $S$ is a prime Jordan ring.

However, *-primeness is still not a necessary condition for $S$ to be prime. Let $A=R \oplus I$, where $R$ is a prime ring with ${ }^{*}$ and $I$ satisfies $I^{2}=(0)$ and $i^{*}=-i$, all $i \in I$. Then $S_{A}$, the symmetric elements of $A$, equals the symmetric elements of $R$, so is prime. Clearly, $A$ is not ${ }^{*}$-prime since $I^{*}=I$ and $I^{2}=(0)$.

To conclude this section, we will prove for prime ideals the analog of Theorem 2 .

THeORem 10. If $R$ is a ring with ${ }^{*}$ and $P$ is a prime ideal of $R$, then $P \cap S$ is a prime Jordan ideal of $S$.

Proof. Consider $P \cap P^{*}$. Since $P$ is prime, $P^{*}$ is also prime. Let $\bar{R}=R /\left(P \cap P^{*}\right)$; since $\left(P \cap P^{*}\right)^{*}=P \cap P^{*}, \bar{R}$ has an induced involution. It is not difficult to check that $\bar{R}$ is *-prime. Let $\bar{S}$ denote the image in $\bar{R}$ of the symmetric elements $S$ of $R$. Though $\bar{S}$ may not be all of the symmetric elements of $\bar{R}, \bar{S}$ satisfies the hypotheses for $T$ in Theorem 9 and, thus, is a prime Jordan ring.

But $\bar{S}=S+\left(P \cap P^{*}\right) /\left(P \cap P^{*}\right) \simeq S /\left(P \cap P^{*} \cap S\right)=S /(P \cap S)$, since $P \cap P^{*} \cap S$ $=P \cap S$. Since $S /(P \cap S)$ is prime, $P \cap S$ is a prime Jordan ideal of $S$.

IV. Further conditions for primeness. Although the $a S b$ condition has the advantage of looking like the condition for primeness of an associative ring, it has the disadvantage that, in general, the element $a s b$, where $a, s$, and $b$ are in $S$, is not a symmetric element. The conditions in the following theorem remedy this problem.

THEOREM 11. If $R$ is 2-torsion free, then the following properties are equivalent. Moreover, for any characteristic, (1) and (2) are equivalent to (5).

(1) $S$ is prime.

(2) $a S b=(0), a, b \in S$ implies $a=0$ or $b=0$.

(3) $s U_{a, b}=0, a, b \in S$ for all $s \in S$ implies $a=0$ or $b=0$ (where $s U_{a, b}=a s b+b s a$ ).

(4) $b U_{s} U_{a}=0, a, b \in S$ for all $s \in S$ implies $a=0$ or $b=0$ (where $b U_{s} U_{a}=a s b s a$ ).

(5) $S U_{b} U_{s} U_{a}=(0)$ for all $s \in S$ implies $a=0$ or $b=0$.

Proof. That (1) and (2) are equivalent has already been done. It is also clear that both (3) and (4) imply (2); thus, it will be enough to show that (2) implies (4) and that (4) implies (3), and that (5) is equivalent to (2). 
We first show that (4) implies (3). Assume that (4) holds but that $a s b+b s a=0$ all $s \in S$ for some $a, b \in S$. We need $a=0$ or $b=0$. Multiplying $a s b+b s a=0$ on the right by $s a$, we see $a s b s a+b s a s a=0$. Multiplying $a s b+b s a=0$ on the left by $a s$ gives $a s a s b+a s b s a=0$. Adding, 2asbsa+asasb+bsasa=0. But $a(\operatorname{sas}) b+b(\operatorname{sas}) a=0$ since $s a s \in S$, and so $2 a s b s a=0$. Thus, asbs $a=0$, and $a=0$ or $b=0$ by property (4).

The more difficult part is in showing that (2) implies (4). Assume that (2) holds but that $a s b s a=0$, all $s \in S$, for some $a, b \in S$. We first see that this implies that $a s b+b s a=0$, all $s \in S$. To show this, by condition (2) it will be enough to show that $(a s b+b s a) t(a s b+b s a)=0$, all $t \in S$.

Linearizing asbsa $=0$, we see that

$$
a s b r a+a r b s a=0, \quad \text { all } r, s \in S .
$$

Thus,

$$
a s b(t b s+s b t) a+a(t b s+s b t) b s a=0, \quad \text { all } s, t \in S
$$

But

$$
a s b(s b t) a+a(t b s) b s a=a(s b s) b t a+a t b(s b s) a=0,
$$

and, thus, 2asbtbsa $=0$, so

$$
\text { asbtbsa }=0, \quad \text { all } s, t \in S .
$$

But then $b s a s b t b s a s b=0$, so since $b s a s b \in S, b s a s b=0$ by condition (2). Going through the same process, we see that

$$
\text { bsatasb }=0, \quad \text { all } s, t \in S .
$$

Multiplying $a t b s a+a s b t a=0$ on the right by $s b$, we have atbsasb $+a s b t a s b=0$. Since $b s a s b=0$, we have $a s b t a s b=0$. By applying *, it is also seen that bsatbsa $=0$. But now

$$
(a s b+b s a) t(a s b+b s a)=a s b t a s b+a s b t b s a+b s a t a s b+b s a t b s a=0,
$$

so that $a s b+b s a=0$, all $s \in S$.

We are now able to show that $a=0$ or $b=0$. For, since $a s b=-b s a$ and $b s a s b=0$, we have $b s a s a=0, b s b s a=0$, asas $b=0$, and $a s b s=0$. Since $a r b s a+a s b r a=0$, it is also true that $b r a s a+a s a r b=0$. Multiplying on the right by $s b$, since $a s a s b=0$, it follows that

$$
\text { asarbsb }=0, \quad \text { all } r, s \in S .
$$

But $a s a, b s b \in S$, so by the $a S b$ condition, for any $s \in S$, as $a=0$ or $b s b=0$. It is well known that, if $f$ and $g$ are linear maps, $S \rightarrow R$ such that for each $s \in S, f(s)=0$ or $g(s)=0$, then $f(s)=0$ for all $s \in S$ or $g(s)=0$ for all $s \in S$. Hence, as $a=0$ for all $s \in S$ or $b s b=0$ for all $s \in S$. Therefore, $a=0$ or $b=0$.

Finally, (5) $\Leftrightarrow(2)$. That (5) $\Rightarrow(2)$ is trivial. Conversely, suppose $a s b t b s a=0$ for all $s, t \in S$. We first show $\{a s b\}=a s b+b s a=0$ by showing $\{a s b\} S\{a s b\}=(0)$. First 
$b s(a s b t b s a) s b=0$, so that $b s a s b=0$. Replacing $t$ by satas, we have asbsatasbs $a=0$, which implies $a s b s a=0$. The usual linearization gives $b s a t b s a=-b$ tasbs $a=0$ for $t \in S$, and $a s b t a s b=0$ by applying *. Finally, bsatasb $=0$ since bsat(asbSbsa)tasb $=(0)$. Therefore, $\{a s b\} S\{a s b\}=(0)$ and by (2), $\{a s b\}=0$. From this, $0=a s b t b s a$ $=-b s a t b s a=b s b t a s a$. By (2), $b s b=0$ or $a s a=0$ for any $s \in S$. Hence, $b s b=0$ for all $s$ or $a s a=0$ for all $s$, and, lastly, $b=0$ or $a=0$.

Unfortunately, the conclusions of Theorem 11 do not hold if the characteristic of $R$ is 2 . It is trivial that if $S$ is prime, (3) need not hold; simply let $R$ be any ring in which $S$ is prime, and let $a=b$; or, let $a$ and $b$ centralize $S$. Condition (4) also fails. For example, let $F$ be a field of characteristic 2 and let $R$ be the $2 n \times 2 n$ matrices over $F$. Let the involution be given by matrix transpose. Then if we let $a=e_{11}$ and

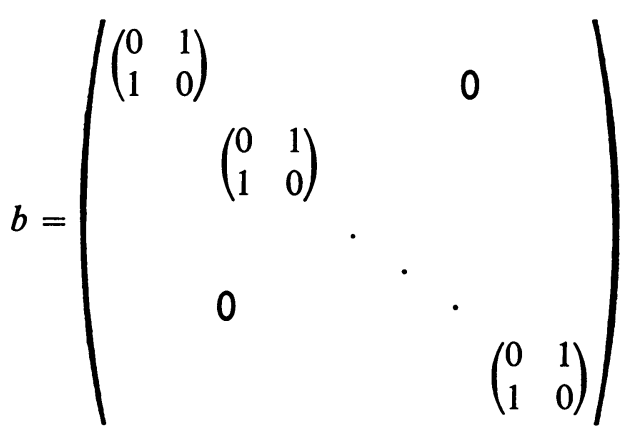

$a s b s a=0$ for all $s \in S$. $S$ is certainly prime in this example since $R$ is prime.

The authors wish to thank the referee for his helpful suggestions.

\section{REFERENCES}

1. N. Jacobson, Structure of rings, Amer. Math. Soc. Colloq. Publ., vol. 37, Amer. Math. Soc., Providence, R. I., 1956. pp. 193-196. MR 18, 373.

2. - Structure theory for a class of Jordan algebras, Proc. Nat. Acad. Sci. U.S.A. 55 (1966), 243-251. MR 33 \#1337.

3. W. S. Martindale III, Rings with involution and polynomial identities, J. Algebra 11 (1969), 186-194. MR 38 \#3302.

4. K. McCrimmon, A general theory of Jordan rings, Proc. Nat. Acad. Sci. U.S.A. 56 (1966), 1072-1079. MR 34 \#2643.

5. - On Herstein's theorems relating Jordan and associative algebras, J. Algebra 13 (1969), 382-392. MR 40 \#2721.

6. - The radical of a Jordan algebra, Proc. Nat. Acad. Sci. U.S.A. 59 (1969), 671-678.

7. C. Tsai, The prime radical in a Jordan ring, Proc. Amer. Math. Soc. 19 (1968), 1171-1175. MR 37 \#6336.

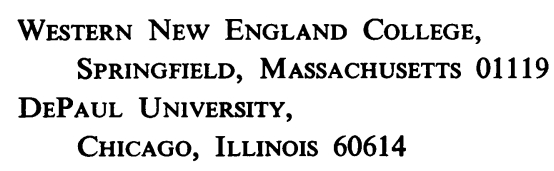

